\title{
La perception des avantages et des contraintes de l'écimage du cotonnier par les producteurs du Mali
}

\author{
Mama Diarra ${ }^{1, *}$, Michel Havard ${ }^{2,3}$ et Mamy Soumaré ${ }^{4}$ \\ ${ }^{1}$ Institut d'économie rurale (IER), Bamako, Mali \\ ${ }^{2}$ Centre de coopération internationale en recherche agronomique pour le développement (CIRAD), UMR Innovation, CIRAD, \\ 34398 Montpellier, France \\ 3 Innovation, Univ. Montpellier, CIRAD, Montpellier, France \\ ${ }^{4}$ IER, Université des sciences sociales et de gestion, Bamako, Mali
}

\begin{abstract}
Résumé - Au Mali, l'efficacité de la lutte chimique systématique (traitements calendaires) contre les ravageurs du cotonnier est régulièrement remise en cause. Des alternatives à cette lutte, comme la lutte étagée ciblée, l'intervention sur seuil et la culture biologique, visant toutes à réduire les quantités de pesticides utilisées, sont diffusées; d'autres sont testées chez les producteurs, comme l'écimage du cotonnier. Cette étude analyse la perception de la technique de l'écimage du cotonnier par des producteurs. Elle a été conduite dans quatre villages de la zone cotonnière, à l'aide de focus groups d'hommes et de femmes et d'entretiens avec 40 producteurs ayant pratiqué ou non l'écimage. Les avantages de l'écimage identifiés par la majorité des producteurs interrogés sont: (i) une technique manuelle facile à mettre en œuvre, (ii) la diminution des risques de chute d'organes fructifères, (iii) la réduction de la hauteur des plants, s'étalant davantage (iv) une amélioration du rendement et (v) la réduction de la population des ravageurs et donc de l'utilisation des pesticides, ayant des effets positifs sur la santé humaine et l'environnement, et diminuant les coûts de production. Ses principales contraintes sont le besoin supplémentaire de maind'œuvre et son coût par rapport à la culture de coton non écimé. Des partenariats avec les sociétés cotonnières et les organisations paysannes et la formation de leurs agents à l'écimage sont indispensables pour une adoption de cette technique à une plus large échelle.
\end{abstract}

Mots clés : perception / écimage / cotonnier / réduction de pesticides / Mali

\begin{abstract}
Perception of advantages and constraints of cotton topping by Mali farmers. In Mali, the effectiveness of systematic chemical control (calendar treatment) against cotton pests is regularly discounted. Some alternatives to this control, aiming at reducing the quantities of pesticides, are being disseminated; others are being tested by producers, such as cotton topping. This study aimed at understanding the perception of the cotton topping technique by farmers. It was conducted through a participatory approach in four villages, using focus groups with men and women, and interviews with about 40 producers who had, or not, practiced topping. Farmers identified the following advantages of cotton topping by: (i) a technique easy to implement by both men and women, (ii) reduction in flower and capsule drop, (iii) reducing the height of plants with more spread out, (iv) for some of them, yield improvement, (v) reducing the population of pests, and therefore the use of pesticides, which has positive effects on human health and the environment, and lower production costs. The main constraint farmers highlighted was the additional need for labor, its higher cost compared to the cultivation of non-topped cotton. Partnerships with cotton companies and farmers' organizations and the training of their agents in cotton topping are essential for a larger scale adoption.
\end{abstract}

Keywords: perception / topping / cotton plant / pest reductions / Mali

\footnotetext{
* Auteur de correspondance : mama5580diarra@gmail.com
} 


\section{Introduction}

L'agriculture malienne occupe plus de $75 \%$ de la population active du pays et contribue pour environ $40 \%$ au produit intérieur brut (PIB) (ODHD, 2016). Le coton représente $62 \%$ des exportations agricoles du pays (Hussein et al., 2005). Une production de 645000 tonnes de cotongraine, payée à un prix garanti aux producteurs de $250 \mathrm{~F} \mathrm{CFA} /$ $\mathrm{kg}(1 €=655,957 \mathrm{FCFA})$, a été enregistrée en 2016/2017 (Diakité et al., 2019). Les avantages économiques et sociaux liés à la culture du coton ne doivent pas cacher des contraintes au champ :

- la pluviométrie aléatoire et inégalement répartie dans le temps et l'espace entraîne pour toutes les cultures de fortes variations de rendement, et pour le coton cela a des conséquences particulièrement fortes sur les revenus monétaires des exploitations;

- le maintien de la fertilité des sols demande une forte production de fumure organique malgré l'accès aux fertilisants chimiques;

- le cotonnier est très sensible aux ravageurs (Guibert et al., 2003 ; Diarra, 2010);

- le rendement du coton graine stagne autour d'une tonne à l'hectare depuis une décennie.

$\mathrm{Vu}$ la forte pression parasitaire, pouvant occasionner des dégâts importants (dessèchement des feuilles, attaque des bourgeons, des fleurs, des capsules, etc.) sur le rendement et la qualité du coton, la lutte chimique systématique (traitement calendaire) est généralisée. Elle consiste en moyenne en six applications d'insecticides tous les 14 jours, du $45^{\mathrm{e}}$ au $115^{\mathrm{e}}$ jour après la levée (Téréta, 2015). Son efficacité sur les populations de ravageurs est régulièrement remise en cause; elle dépend du nombre de traitements, des doses utilisées, de la technicité des producteurs et des résistances aux pesticides acquises par les ravageurs. Ces résistances font que les pesticides doivent être régulièrement changés pour maintenir leur efficacité (Haubruge et Amichot, 1998; Brévault et al., 2003 ; Jourdain et Perrin, 2014). L'utilisation de pesticides présente des risques pour la santé des agriculteurs et tue les insectes non nuisibles présents sur la parcelle au moment des traitements. Des alternatives à la lutte chimique visant la réduction de l'utilisation des pesticides sont diffusées mais peu adoptées; en 2008, sur les 521000 ha cultivés, les principaux modes de protection du coton étaient le traitement calendaire régulier sur 431600 ha (82\%), la lutte étagée ciblée (LEC) sur 71800 ha $(14 \%)$, le traitement sur seuil (TS) sur 15100 ha (3\%), la gestion intégrée de la production et des déprédateurs (GIPD) sur 1500 ha $(0,3 \%)$ et la culture biologique (CB) sur 930 ha $(0,2 \%)$ (Bagayoko, 2013). Une autre méthode possible de contrôle des ravageurs est l'écimage, elle était préconisée au début du $\mathrm{xx}^{\mathrm{e}}$ siècle pour limiter les populations de chenilles de la capsule (Vayssières et Mimeur, 1926). Mais l'écimage a été progressivement abandonné par les producteurs en absence d'impact perceptible sur les rendements, alors qu'il augmente la quantité de travail. La recherche sur l'écimage a repris en 2002 au Mali, suite au constat de la faible participation à la production finale des organes fructifères produits après l'apparition de la $15^{\mathrm{e}}$ branche fructifère (Renou et al., 2016). Téréta (2015, p. 137) a proposé de relancer cette pratique: «Par le passé, des producteurs de coton au Mali ont écimé leurs cotonniers. Mais ils ont progressivement abandonné cette pratique en raison de l'absence de régularité d'apparition de ses avantages agronomiques et productifs. Nous faisons le même constat mais nous recommandons toutefois aux producteurs de pratiquer de nouveau l'écimage de cotonniers de façon raisonnée en considérant surtout les avantages phytosanitaires qu'il procure: un bon contrôle des chenilles de la capsule qui réduit l'utilisation d'insecticides, augmente leurs revenus, garantit une meilleure durabilité de la culture, préserve l'environnement et diminue les risques en santé humaine». Suite aux bons résultats obtenus en station de recherche (réduction de la quantité de pesticide utilisée, caractère répulsif des ravageurs par la sève, pâleur éclatante de la fibre du coton) (Renou et al., 2011), l'écimage du cotonnier est testé depuis 2014 dans quelques villages de la zone cotonnière. La pratique spontanée de l'écimage par de nombreux producteurs en plus de ceux appuyés par les conseillers agricoles dans les villages a été à l'origine du questionnement de cette étude sur leur perception de l'écimage. Celui-ci consiste à couper la partie du bourgeon terminal de la tige principale au $65^{\mathrm{e}}$ jour du cycle végétatif pour le protéger contre les agressions (Renou et al., 2012). Il est réalisé manuellement, en pinçant les tissus tendres du bourgeon terminal d'un cotonnier entre les $3^{\mathrm{e}}$ et $4^{\mathrm{e}}$ feuilles terminales en partant du sommet du plant et en le sectionnant par pression et torsion. Il permet de réduire le nombre de traitements chimiques après sa réalisation (Renou et al., 2011, 2012; Téréta, 2015).

Cet article analyse la perception de la technique de l'écimage du cotonnier par les producteurs maliens. Il décrit la méthodologie utilisée et présente les résultats sur les caractéristiques, la place du coton et la pratique de l'écimage du cotonnier dans les exploitations agricoles. Puis, il discute les indicateurs de la perception des producteurs de l'écimage et conclut sur des propositions pour favoriser la diffusion de l'écimage du cotonnier.

\section{Méthodologie}

\subsection{La démarche mise en œuvre}

La méthode mixte d'analyse reposant sur l'intégration, la collecte et l'analyse de données quantitatives et qualitatives est mobilisée (Small, 2011). Les données collectées permettent de renseigner des indicateurs de la perception qui sont des éléments ou des signes à travers lesquels la cause de l'acceptation ou du rejet d'une idée, d'un projet, etc. par un individu ou un groupe d'individus peut être comprise (Couty et Winter, 1983; Peersman, 2014). La perception est définie comme un jugement porté par un utilisateur sur la technologie proposée et sur la manière de la mettre en œuvre.

La triangulation, c'est-à-dire l'utilisation de manière combinée de différentes techniques de récolte des données, est utilisée (Denzin, 2009). Elle permet d'avoir une connaissance plus complète et différenciée sur le phénomène étudié dans le cadre d'une approche de recherche essentiellement qualitative avec un nombre limité d'informations collectées.

La perception des producteurs des avantages et des contraintes de l'écimage du cotonnier a été appréhendée à 


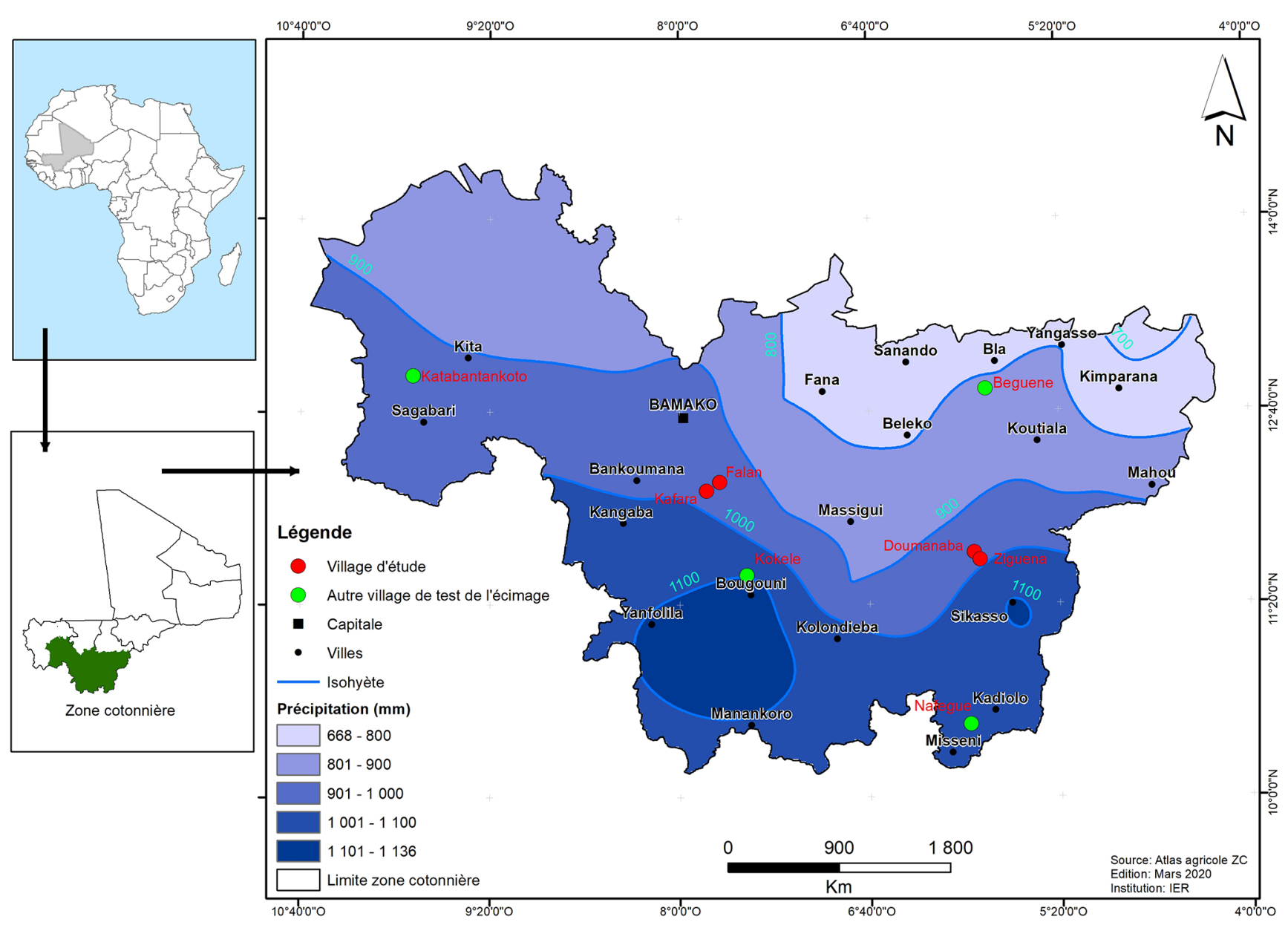

Fig. 1. Délimitation de la zone cotonnière et localisation des villages de l'étude.

Fig. 1. Delimitation of the cotton zone and location of the study villages.

l'aide d'indicateurs agronomiques, économiques, sociaux et environnementaux identifiés par l'équipe de recherche et par les producteurs. Ces indicateurs expriment des avantages de l'écimage :

- la réduction de la hauteur des plants de cotonnier;

- la diminution des risques de chute d'organes fructifères;

- la précocité et l'augmentation de la production, à travers une meilleure rétention des organes fructifères;

- un poids moyen des capsules plus élevé ;

- la diminution de la population des ravageurs (Renou et al., 2012).

Ils expriment aussi des contraintes : le besoin supplémentaire de main-d'œuvre et son coût par rapport à la culture de coton non écimé.

\section{2 Échantillonnage et collecte des données}

Parmi les six villages choisis dans les différentes zones de production du coton au Mali par l'Institut d'économie rurale (IER) et les sociétés cotonnières (Fig. 1), deux, Kafara et Ziguéna, ont été choisis pour cette étude réalisée en 2016 (Diarra, 2016), car les travaux sur l'écimage y sont menés depuis 2014. Deux autres villages, où l'écimage a été adopté par certains producteurs et situés dans un rayon de $20 \mathrm{~km}$ de chacun des précédents, ont aussi été ajoutés à l'échantillon : Falan et Doumanaba (Fig. 1). Les villages de Kafara et Falan sont situés dans la zone de l'Office de la haute vallée du Niger (OHVN) ; la pluviométrie moyenne se situe autour de $800 \mathrm{~mm}$. Les villages de Ziguéna et Doumanaba sont situés dans la zone de la Compagnie malienne de développement des textiles (CMDT) de Sikasso-Bougouni, la pluviométrie moyenne annuelle y est de $1000 \mathrm{~mm}$.

Les données ont été collectées auprès d'hommes et de femmes, avec les techniques suivantes:

- treize focus groups: 9 groupes d'hommes, dont 3 à Kafara (19 hommes), 2 à Falan (16 hommes), 3 à Ziguéna (21 hommes), 1 à Doumanaba ( 8 hommes), quatre de femmes, dont 2 à Kafara (17 femmes), 2 à Ziguéna (13 femmes); les discussions ont porté sur leurs informations, leurs connaissances et leurs pratiques de l'écimage, mais aussi sur les avantages et les inconvénients de cette technique ;

- des entretiens individuels avec 40 producteurs (soit $30 \%$ du total) des villages de Kafara et Ziguéna ( 20 expérimentateurs « EXP» ayant testé l'écimage avec la recherche et 20 non-expérimentateurs «NEXP» ne l'ayant pas testé avec la recherche); les entretiens ont porté sur les structures de leurs exploitations, sur leurs pratiques, sur 
Tableau 1. Perception de l'écimage par les 40 producteurs de l'échantillon.

Table 1. Perception of cotton topping by the 40 sampled farmers.

\begin{tabular}{llll}
\hline Réponses producteurs & \% échantillon & \% EXP & \% NEXP \\
\hline Augmentation du revenu d'exploitation & 63 & 65 & 61 \\
Coût abordable & 55 & 70 & 40 \\
Efficacité contre les ravageurs & 53 & 83 & 23 \\
Augmentation du rendement du coton & 10,5 & 9 & 12 \\
Gain de temps sur les traitements & 9,5 & 13 & 6 \\
Effet positif sur la santé humaine & 9,5 & 4 & 6 \\
Facile à réaliser & 5 & 4,2 & 6 \\
Disponibilité de la main-d'œuvre & 2,1 & 0 & 0 \\
Sans avis & 18 & 36 \\
\hline
\end{tabular}

EXP: expérimentateur; NEXP: non-expérimentateur.

Source : Diarra, 2016.

la lutte contre les ravageurs du cotonnier et sur les avantages et inconvénients de l'écimage;

- des entretiens avec deux techniciens de la recherche dans le village de Kafara et avec quatre dans celui de Ziguéna.

\subsection{Analyse et traitement des données}

Les données quantitatives des entretiens individuels avec les producteurs ont été analysées avec des statistiques descriptives (sommes, moyennes, pourcentages) à l'aide du tableur Excel. Elles ont permis de caractériser la place et l'importance du coton, la mobilisation de la main-d'œuvre et des équipements pour les différentes activités des exploitations agricoles de l'échantillon.

Les entretiens en focus groups ont été enregistrés avec un dictaphone, puis retranscrits en verbatim après écoute, et enfin synthétisés et analysés. Ils ont permis d'identifier les avantages et inconvénients (agronomiques, économiques, sociaux et environnementaux) de l'écimage du cotonnier perçus par les producteurs.

\section{Résultats}

\subsection{Pratique généralisée de la lutte chimique contre les ravageurs du cotonnier dans les exploitations agricoles}

Tous les chefs d'exploitation de l'échantillon sont des hommes; deux tiers ont plus de 45 ans, moins de $20 \%$ ont été à l'école jusqu'au primaire. Les exploitations, souvent composées de plusieurs ménages, comptent en moyenne 18 personnes, dont 7 actifs parmi lesquels une moitié de femmes. Le nombre d'actifs familiaux est important pour la réalisation des travaux agricoles, en particulier l'écimage du cotonnier, généralement réalisé par les femmes et les enfants scolarisés (3 en moyenne par exploitation).

La superficie moyenne cultivée par exploitation est de 15 ha: $45 \%$ de coton, $23 \%$ de maïs, $18 \%$ de sorgho, $7 \%$ de niébé, $3 \%$ de mil, $3 \%$ de riz et $1 \%$ d'arachide. Le coton est cultivé en culture pure, car l'utilisation d'herbicides et d'insecticides ne permet pas d'y associer d'autres cultures.
Les fumures minérales appliquées sur coton sont l'engrais complexe NPKSB (14-18-18-6-1, $145 \mathrm{~kg} / \mathrm{ha}$ ) et l'urée (46-060, $50 \mathrm{~kg} / \mathrm{ha})$. Toutes les exploitations utilisent la traction animale pour les travaux du sol, les semis, l'entretien des cultures (sarclage, buttage) et les transports, et trois possèdent un tracteur. $98 \%$ des exploitations utilisent les insecticides des six traitements de la lutte chimique calendaire sur coton, $90 \%$ la fumure organique, $65 \%$ les herbicides totaux, $56 \%$ les herbicides sélectifs. Sur coton, $80 \%$ des producteurs utilisent la traction animale pour les sarclages et le buttage, et $52 \%$ pour le semis. Le coton occupe la première ou la deuxième place dans les revenus de plus de $95 \%$ des exploitations agricoles. Les autres sources de revenus sont l'élevage, le commerce et l'artisanat.

\subsection{Les producteurs perçoivent les avantages et inconvénients techniques, économiques et environnementaux de l'écimage}

Les discussions (focus groups) des hommes font ressortir que l'écimage du cotonnier a été introduit pour améliorer le rendement à Kafara et Falan par des migrants de Ségou et de Côte d'Ivoire depuis une quarantaine d'années. Il était réalisé à la main, au bâton, avec une branche de taille moyenne, accidentellement par les bœufs en divagation ou lors des travaux de buttage et de sarclage. L'écimage du cotonnier permettait d'obtenir plus de branches fructifères, mais il n'a pas été adopté car il demandait un travail supplémentaire jugé trop pénible.

Les discussions (focus groups) des femmes pratiquant l'écimage du cotonnier montrent que les femmes apprécient la technique de l'écimage de $100 \%$ des plants de cotonnier, jugée facile à réaliser, et efficace contre les ravageurs et pour l'amélioration du rendement. Mais, les femmes ont émis des doutes sur l'efficacité de l'écimage limité à $20 \%$ des plants de cotonnier. Pour elles, ce sont les chefs d'exploitation qui vont décider ou non de continuer l'écimage du cotonnier.

Les entretiens individuels avec les 40 producteurs de l'échantillon montrent que les principaux avantages de l'écimage sont une augmentation du revenu, un coût abordable et l'efficacité contre les ravageurs; ces avantages sont mieux perçus par les producteurs EXP (Tab. 1). Ce sont surtout les 
Tableau 2. Perception de la technique de l'écimage du cotonnier lors des focus groups.

Table 2. Perception of the cotton topping during focus groups.

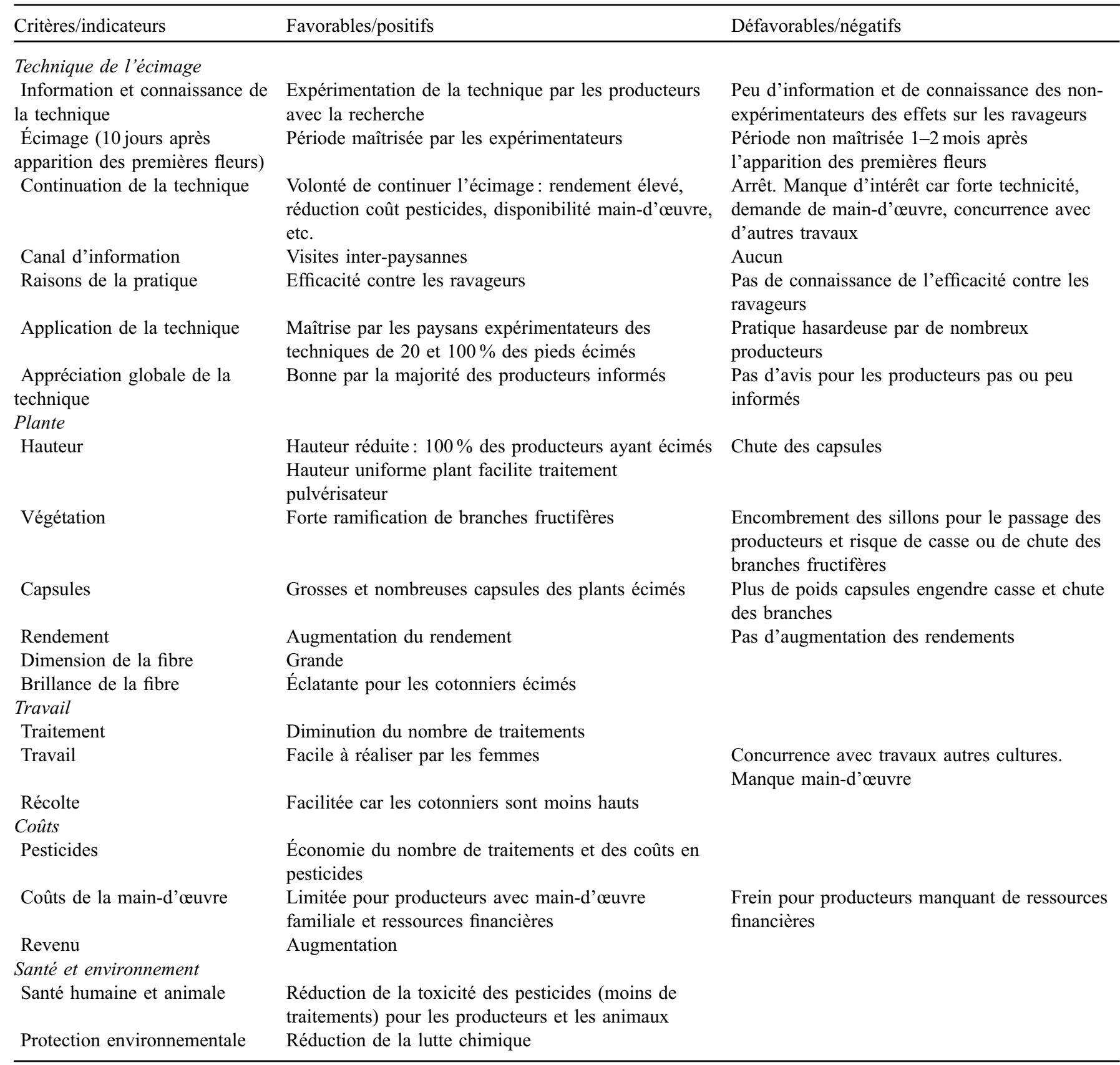

Source : Diarra, 2016.

femmes et les enfants qui éciment, car c'est un travail facile et peu physique. Mais c'est un travail supplémentaire dans un calendrier agricole chargé et un travail technique nécessitant l'observation des règles, en particulier le respect scrupuleux de la date d'écimage et de la partie à écimer. L'économie de pesticides permise par l'écimage est mise en avant, avec des effets positifs sur la santé humaine. Un tiers des producteurs NEXP n'étant pas familiers de l'écimage n'ont pas donné d'avis.

Les indicateurs de perception de l'écimage du cotonnier par les différents focus groups portent sur la technique de l'écimage, la plante, le travail, les coûts, la santé et l'environnement (Tab. 2).

Les entretiens avec les techniciens ressortent les limites à la diffusion à grande échelle de l'écimage :

- pénibilité de la technique;

- faible couverture en techniciens et en producteurs EXP susceptibles de transmettre leurs connaissances ;

- demande en main-d'œuvre à une période où cette demande est forte dans les exploitations ;

- insuffisance de communication et d'information : émissions radio, projections vidéo, dépliants en langue locale, etc. 


\section{Discussions}

La place du coton dans les exploitations agricoles et les indicateurs de perception de l'écimage sont les éléments majeurs dans le choix des producteurs de pratiquer ou non l'écimage.

Lors des focus groups, les indicateurs montrant les avantages (efficacité contre les ravageurs, économie sur les pesticides, facile à pratiquer par les femmes, etc.) de l'écimage sont apparus déterminants dans leur choix de continuer sa pratique et dans l'intérêt des villages voisins pour accueillir des expérimentations sur l'écimage du cotonnier.

Le respect de la période d'écimage est un point important mis en avant par les producteurs, ce qui est en cohérence avec les résultats des travaux expérimentaux sur l'écimage (Renou, 2016). Les producteurs mentionnent des effets de l'écimage sur la plante (réduction de la hauteur, ramification du plant, grosseur et nombre élevé de capsules, éclat de la fibre du coton, etc.) déjà observés dans les expérimentations de la recherche (Renou, 2016). Les producteurs constatent aussi une réduction du nombre de traitements insecticides, donc de la quantité de pesticides utilisée et du coût associé.

Le besoin de main-d'œuvre pour réaliser l'écimage est différemment perçu par les producteurs. Pour ceux ayant de la main-d'œuvre disponible et/ou les moyens de payer de la main-d'œuvre salariée, la pratique de l'écimage s'insère dans le calendrier agricole et permet de réduire les coûts de production du coton. Pour ceux disposant de peu de maind'œuvre et de faibles ressources financières, réaliser l'écimage à une période intense de travaux manuels (entretien de toutes les cultures pluviales) est une contrainte forte à la pratique de l'écimage et au respect des périodes favorables. Selon les producteurs, l'écimage demande plus de temps ( 3 à 6 jours/ha) que le traitement chimique ( 2 à $4 \mathrm{~h} / \mathrm{ha}$ ). Pour la majorité des producteurs, l'écimage n'améliore pas les rendements du coton, seuls quelques-uns annoncent des augmentations de rendement de 100 à $200 \mathrm{~kg} / \mathrm{ha}$. Les expérimentations de la recherche montrent en moyenne une amélioration de rendement en coton graine de $15,1 \%$ sur les parcelles écimées à $100 \%$ et de $9 \%$ sur celles écimées à $20 \%$ par rapport aux parcelles où les traitements insecticides sont pratiqués (Renou, 2016).

La majorité des producteurs mentionnent une réduction des coûts de production sur les parcelles écimées, liée aux économies sur les appareils de traitement et leurs consommables (piles) et sur les produits insecticides, du fait de la diminution du nombre de traitements (réduction de deux bidons de pesticides par hectare soit une économie monétaire de 10000 F CFA par hectare).

La perception des producteurs est en lien avec l'intérêt qu'ils tirent de la mise en œuvre de l'écimage du cotonnier (augmentation du rendement et réduction de la quantité de pesticide achetée). Pour certains, écimer est très technique et sa réalisation est contraignante, comme le respect de la date d'écimage 10 jours après l'apparition des premières fleurs soit au $65^{\mathrm{e}}$ jour après la levée (Nonfon et Hinvi, 2014). En effet, certains producteurs éciment entre 40 et 50 jours après les semis (Renou, 2016).

\section{Conclusion}

Les indicateurs de perception de l'écimage du cotonnier par les producteurs concernent différents éléments de la conduite de la culture cotonnière :

- la maitrise et la faisabilité de la technique de l'écimage ; - ses effets sur la plante, sur la santé humaine, sur l'environnement et sur le travail au sein de l'exploitation; - ses résultats sur les coûts de production.

Les avantages de l'écimage (agronomiques, économiques, sociaux, environnementaux) identifiés par les producteurs sont :

- une technique manuelle facile à mettre en œuvre;

- la diminution de la chute des organes fructifères;

- la réduction de la hauteur des plants, s'étalant davantage ;

- l'amélioration du rendement pour certains producteurs;

- la réduction de l'utilisation des pesticides ayant des effets positifs sur la santé humaine, sur l'environnement, et diminuant les coûts de production.

Les principales contraintes exprimées par les producteurs sont la réalisation de l'écimage à une date précise et l'augmentation du travail au sein de l'exploitation, à une période où d'autres travaux sont à faire.

Cependant, bien que certains producteurs envisagent de continuer à pratiquer l'écimage, on ne peut pas conclure avec certitude sur l'adoption définitive de l'écimage par ces derniers, ni sur ses chances de diffusion rapide. En effet, nos résultats doivent être considérés avec prudence, puisque obtenus dans seulement quatre villages; une étude de l'écimage plus étendue sur la zone cotonnière du Mali serait nécessaire pour valider les résultats obtenus et les propositions de cette étude à une plus large échelle.

Avec cette limite, si une diffusion et une adoption à plus grande échelle de l'écimage du cotonnier s'avèrent souhaitables, il serait nécessaire de multiplier les parcelles expérimentales dans les nombreux villages de la zone cotonnière et de mener d'autres actions d'appui-conseil mais aussi de développer les partenariats entre les acteurs du secteur (organisations non gouvernementales, organisations paysannes, sociétés cotonnières, etc.).

Remerciements. Les auteurs remercient l'Agence française de développement (AFD) qui a financé le projet d'Appui à l'amélioration de la gouvernance de la filière coton dans sa nouvelle configuration institutionnelle à la productivité et à la durabilité des systèmes d'exploitation en zone cotonnière (PASE II) support à la réalisation de cette étude.

\section{Références}

Bagayoko K. 2013. L'importance et l'avenir du coton en Afrique de l'Ouest: cas du Mali. Thèse en sciences économiques. France: université de Grenoble. https://tel.archives-ouvertes.fr/tel00959321/document

Brévault T, Béyo J, Nibouche S, Vayssières M. 2003. La résistance des insectes aux insecticides : problématique et enjeux en Afrique 
centrale. In : Jamin JY, Seiny Boukar L, Floret C, eds. Savanes africaines : des espaces en mutation, des acteurs face à de nouveaux défis. Actes du colloque, Garoua, Cameroun, 27-31 mai 2002. http://agritrop.cirad.fr/509790/

Couty P, Winter G. 1983. Qualitatif et quantitatif, deux modes d'investigation complémentaires. Réflexions à partir des recherches en milieu rural africain. Paris: ORSTOM, AMIRA, brochure 43, 78 p. https://horizon.documentation.ird.fr/exl-doc/pleins_tex tes/divers4/15287.pdf

Denzin NK. 2009. The research act: a theoretical introduction to sociological methods, 1st ed. New York: Routledge. https://doi.org/ $10.4324 / 9781315134543$

Diakité M, Soumaré M, Traoré S. 2019. Dynamique récente et perspective de développement du système coton au Mali. In : Soumaré M, ed. Atlas des zones cotonnières du Mali, $2^{\mathrm{e}}$ éd. Bamako: Institut d'économie rurale, pp. 40-41. ISBN 978-99952-56-99.

Diarra M. 2016. Perception des producteurs de la technique de l'écimage du cotonnier au Mali : cas des villages de Kafara et Ziguéna. Mémoire de Master International en Innovation et Développement Rural (Agrinovia). Burkina Faso: université Joseph Ki-Zerbo, Ouagadougou I, 71 .

Diarra N. 2010. Étude sur le financement de l'agriculture et du monde rural. Rapport complémentaire. Analyse des filières agricoles: coton, riz, mangue, pomme de terre, échalote. Mali. Rome, Italie: FAO, FARM, crédit Agricole, 49 p. https://www.fondation-farm. org/IMG/pdf/microfi20100701_offre_et_demande.pdf

Guibert H, M'Biandoun M, Olina JP. 2003. Productivité et contraintes des systèmes de culture au Nord-Cameroun. In : Jamin JY, Seiny Boukar L, Floret C, eds. Savanes africaines : des espaces en mutation, des acteurs face à de nouveaux défis. Actes du colloque, Garoua, Cameroun, 27-31 mai 2002. http://agritrop. cirad.fr/509790/

Haubruge E, Amichot M. 1998. Les mécanismes responsables de la résistance aux insecticides chez les insectes et les acariens. Biotechnol. Agron. Soc. Environ. 2(3): 161-174.

Hussein K, Perret C, Hitimana L. 2005. Importance économique et sociale du coton en Afrique de l'Ouest: rôle du coton dans le développement, le commerce et les moyens d'existence. Paris: Secrétariat du Club du Sahel et de l'Afrique de l'Ouest, OCDE, 72 p. http://www.hubrural.org/IMG/pdf/csao_etude_socio_eco_co ton_fr.pdf

Jourdain F, Perrin Y, eds, 2014. Utilisation des insecticides et gestion de la résistance. Montpellier / France: centre IRD / Centre national d'expertise sur les vecteurs (CNEV), 71 p. https://www.anses.fr/fr/ system/files/CNEV-Ft-Fev2014-Rapport_Utilisation_insecticides_ gestion_resistance.pdf

Nonfon $\overline{C R}$, Hinvi JC. 2014. Effet de la période d'écimage sur la productivité du cotonnier biologique dans la commune de OuassaPéhunco. In : Rapport d'activité 2013. Cotonou, Bénin: Institut national des recherches agricoles du Bénin, pp. 38-44.

ODHD. 2016. Rapport national sur le développement humain, édition 2016, Migration, développement et lutte contre la pauvreté. Mali: ODHD, 126 p. http://hdr.undp.org/sites/default/files/rndh_edi tion_2016_version_du_30_mai_2016.pdf

Peersman G. 2014. Présentation des méthodes de collecte et d'analyse de données dans l'évaluation d'impact. Note méthodologique évaluation d'impact $n^{\circ} 10$. Florencia, Italia: Centre de recherche Innocenti de 1'UNICEF, 24 p. https://www.unicef-irc.org/publica tions/pdf/brief_10_data_collection_analysis_fre.pdf

Renou A. 2016. Rapport de recherche, campagne 2015-2016. Activités. Stratégies de protection intégrée de la culture cotonnière. $22^{\mathrm{e}}$ session du Comité de programme. Bamako, Mali, IER, volet R/ D PASE II, $175 \mathrm{p}$.

Renou A, Téréta I, Togola M. 2011. Manual topping decreases bollworm infestations in cotton cultivation in Mali. Crop Protection 30: 1370-1375.

Renou A, Togola M, Téréta I, Brévault T. 2012. First steps towards "Green" cotton in Mali. Outlooks on Pest Management 23(4) : $173-176$.

Renou A, Téréta I, Togola M, Brévault T, Goebel FR, Jean J. 2016. Écimage manuel des cotonniers. Fiche technique. Montpellier, France: CIRAD. https://coton-innovation.cirad.fr/content/down load/4856/35361/file/ITKInnovation-14-Mali\%20Ecimage.pdf

Small ML. 2011. How to conduct a mixed methods study: recent trends in a rapidly growing literature. Annual Review of Sociology 37: $57-86$.

Téréta I. 2015. Contribution à l'amélioration de la protection phytosanitaire de la culture cotonnière au Mali : effets de l'écimage manuel sur les populations des chenilles de la capsule. Thèse de doctorat en sciences biologiques appliquées, spécialité entomologie agricole. Mali: université des sciences, des techniques et des technologies de Bamako. 169 p. https://agritrop.cirad.fr/578097/1/ theseITereta.pdf

Vayssières M, Mimeur J. 1926. Les insectes nuisibles au cotonnier en Afrique occidentale française. Paris: Librairie Emile Larose, bibliothèque de l'Institut d'agronomie coloniale, $175 \mathrm{p}$.

Citation de l'article : Diarra M., Havard M., Soumaré M. 2020. La perception des avantages et des contraintes de l'écimage du cotonnier par les producteurs du Mali. Cah. Agric. 29: 10. 